\title{
Fixed Boundary Vibration Measurement Method for Surface Tension of Membrane Structure
}

\author{
Shen Hao ${ }^{1}$, Xu Xiaochen ${ }^{2}$, Chen Tao ${ }^{1}$, Xu Zhihong ${ }^{1, *}$ \\ ${ }^{1}$ School of Science, Nanjing University of Science and Technology, Nanjing, China \\ ${ }^{2}$ Nanjing Institute of Electronic Technology, Nanjing, China
}

\section{Email address:}

18362903183@163.com (Shen Hao), xuzh a mail.njust.edu.cn (Xu Zhihong)

${ }^{*}$ Corresponding author

\section{To cite this article:}

Shen Hao, Xu Xiaochen, Chen Tao, Xu Zhihong. Fixed Boundary Vibration Measurement Method for Surface Tension of Membrane Structure. Science Discovery. Vol. 7, No. 6, 2019, pp. 390-397. doi: 10.11648/j.sd.20190706.13

Received: November 2, 2019; Accepted: December 3, 2019; Published: December 9, 2019

\begin{abstract}
At present, some methods for measuring the surface tension of membrane structure are applied to engineering practice. But there are some defects happened in the engineering practice. This paper proves the partial boundary of the fixed membrane surface. The natural vibration frequency of the membrane surface is only related to the membrane surface tension. Based on this principle, a magnetic clip-type membrane surface fixed boundary fixture is designed. A method for measuring the tension of the membrane surface tension by measuring the natural vibration frequency of the inner membrane surface of the fixed boundary is proposed. The finite element method was used to analyze the vibration frequency of the fixed boundary membrane under different tensions. The vibration frequency of the fixed boundary membrane under different tensions was measured by experiments. The simulation results were compared with the experimental results, and the accuracy of the method was verified. Through this method, the calibration result of the small sample on the calibration table can be used for the tension measurement of any boundary condition in the actual project and the material film structure, which is in accordance with the requirements of simple operation, wide applicability and high measurement precision in engineering practice.
\end{abstract}

Keywords: Tensioned Membrane Structures, Tension Measurement, Vibration Method, Numerical Simulation

\section{膜结构膜面张力的固定边界振动测量法}

\author{
沈昊 ${ }^{1}$, 徐晓晨 ${ }^{2}$, 陈涛 ${ }^{1}$, 徐志洪 ${ }^{*}$ \\ 1南京理工大学理学院, 南京, 中国 \\ 2南京电子技术研究所, 南京, 中国
}

邮箱

18362903183@163.com (沈吴), xuzh a mail.njust.edu.cn（徐志洪)

\begin{abstract}
摘要: 当前测量膜结构膜面张力的方法应用于工程实际时存在缺陷, 本文经过理论推导, 证明了固定膜面的部分边界, 此边界内膜面自振频率只与膜面张力有关。基于此原理, 设计了一种磁夹式的膜面固定边界夹具, 提出一种通过测量 固定边界内膜面的自振频率，反推出膜面张力的张力测量方法。采用有限元方法分析了不同张力下的固定边界膜面振 动频率, 通过试验测量了不同张力下的固定边界膜面振动频率, 并将仿真结果与试验结果进行比较, 验证了该方法准 确性。通过该方法, 在标定台上对小试样的标定结果, 可用于实际工程中的任意边界条件同材料膜结构的张力测量, 符合工程实际中的操作简单, 适用性广和测量精度高的要求。
\end{abstract}




\section{1. 引言}

张拉膜结构因其新颖的造型和优秀的力学性能, 在结 构中得到广泛应用[1]。2000年后, 膜结构在我国各类体育、 展览、文化等建筑中开始得到迅速的发展和应用[2]。膜结 构在施加预应力后获得一定的承载能力, 为了使膜结构的 承载能力达到设计要求, 需要对膜结构的预张力进行测量。 Reutskiy提出了一种任意形状非均匀膜自由振动问题的数 值方法 [3], 魏德敏和杨桂通用空间有限元、时间差分法分 析了受冲击荷载的矩形固支板的动力响应[4], 邓梁波和叶 天麒获得了固支圆板非线性动力响应的近似解 [5], 江松青 和李永池等讨论了加强筋参数对加筋板在横向冲击载荷 作用下的非线性动力响应和结构效率的影响[6], 国内外学 者提出了“拟索法”、“超声法”和“弹射法”等方法[7 9]。“拟 索法”需要测试区域成为真空环境, 工程中由于工况复杂, 操作过于复杂。“超声法”因为膜结构自身的材料特性, 导 致误差较大。“弹射法”要求采集碰撞小球的入射速度及回 弹速度, 在大型工程中, 由于发射器与膜面的距离不同, 小球的入射速度无法标定。

现有研究证明随着预张力增大, 膜结构的自振频率随 之明显增大 [10], 考虑到膜面边界对自振频率的影响, 本 文通过固定膜面的测量边界, 设计出适用于工程中的膜结 构预张力振动测试方法。

\section{2. 等应力圆形薄膜固有频率近似计算}

运用分离变量法求解等应力薄膜的固有频率 [11]。取 Oxy平面与薄膜变形前所处的平面一致, 薄膜沿 $\mathrm{z}$ 轴的位移 记作 $\mathrm{W}(\mathrm{x}, \mathrm{y}, \mathrm{t})$, 薄膜单位弧长上的张力记作 $\mathrm{T}[12]$ 。

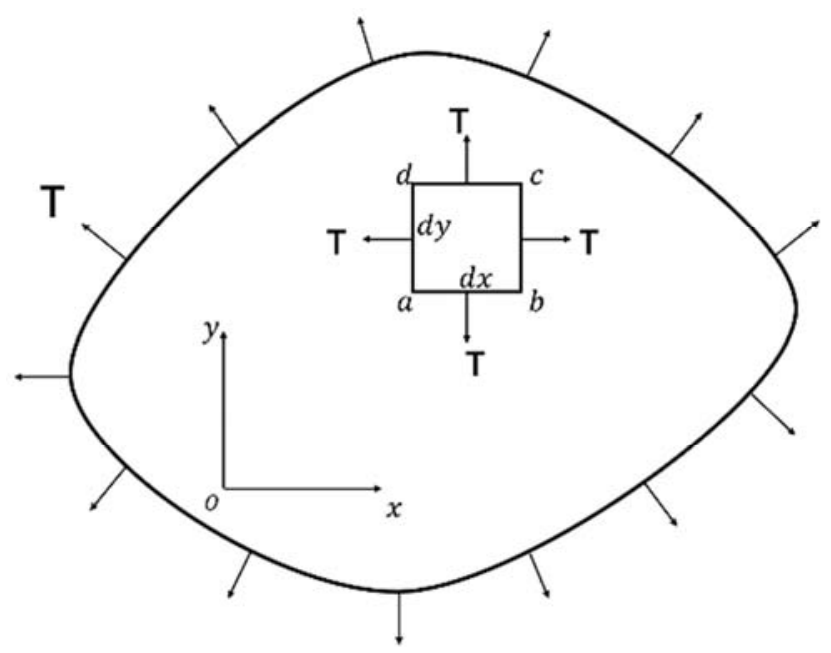

(a)

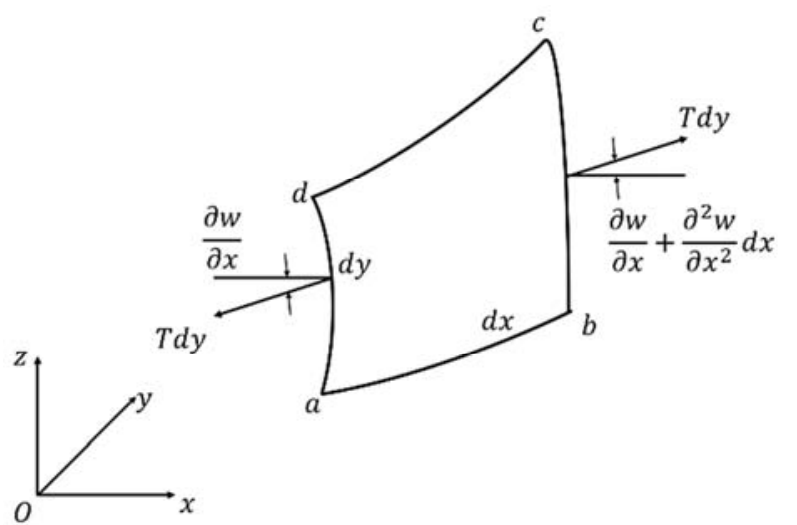

(b)

图1 矩形微元。

考察图1中矩形微元abcd周边承受的张力沿z方向的 合力[13]。微元挠曲后如图1（b）所示, 作用在 $a d 、 b c$ 边 上的张力沿z方向的合力为

$$
T d y\left(\frac{\partial W}{\partial x}+\frac{\partial^{2} W}{\partial x^{2}} d x\right)-T d y \frac{\partial W}{\partial x}=T \frac{\partial^{2} W}{\partial x^{2}} d x d y
$$

同样, 作用在 $a b 、 c d$ 边上张力沿 $z$ 方向的合力为

$$
T \frac{\partial^{2} W}{\partial y^{2}} d x d y
$$

由此可得, 作用在微元abcd四周的张力引起z反方向 的合力为

$$
\mathrm{T}\left(\frac{\partial^{2} W}{\partial x^{2}}+\frac{\partial^{2} W}{\partial y^{2}}\right) d x d y
$$

设薄膜单位面积的质量为常量 $\rho$, 沿 $z$ 方向的干扰力面 密度为 $\mathrm{f}(x, y, t)$, 根据牛顿第二定律得到

$$
T\left(\frac{\partial^{2} W}{\partial x^{2}}+\frac{\partial^{2} W}{\partial y^{2}}\right) d x d y+f d x d y=\rho \frac{\partial^{2} W}{\partial t^{2}} d x d y
$$

化简后便得受迫振动方程

$$
\rho \frac{\partial^{2} W}{\partial t^{2}}-T\left(\frac{\partial^{2} W}{\partial x^{2}}+\frac{\partial^{2} W}{\partial y^{2}}\right)=f
$$

令右端的干扰力 $f$ 为零, 得到自由振动方程

$$
\frac{\partial^{2} W}{\partial x^{2}}+\frac{\partial^{2} W}{\partial y^{2}}=\frac{1}{c^{2}} \frac{\partial^{2} W}{\partial t^{2}}
$$

其中

$$
\mathrm{c}=\sqrt{\frac{T}{\rho}}
$$

式（6）是二维波动方程。讨论固有振动时, 挠度函 数可表示为

$$
W(x, y, t)=W(x, y) \sin (\omega t+\gamma)
$$


将式（8）代入自由振动方程（6）, 得到

$$
\frac{\partial^{2} W}{\partial x^{2}}+\frac{\partial^{2} W}{\partial y^{2}}+\frac{\omega^{2}}{c^{2}} W=0
$$

讨论圆形薄膜的振动问题时, 采用极坐标进行分离变 量会出现贝塞尔 (Bessel) 方程[14]。考虑图2所示的固定 边界的圆形薄膜, 半径为 $l$, 采用极坐标 $(r, \theta)$ 进行求解, 边界条件为

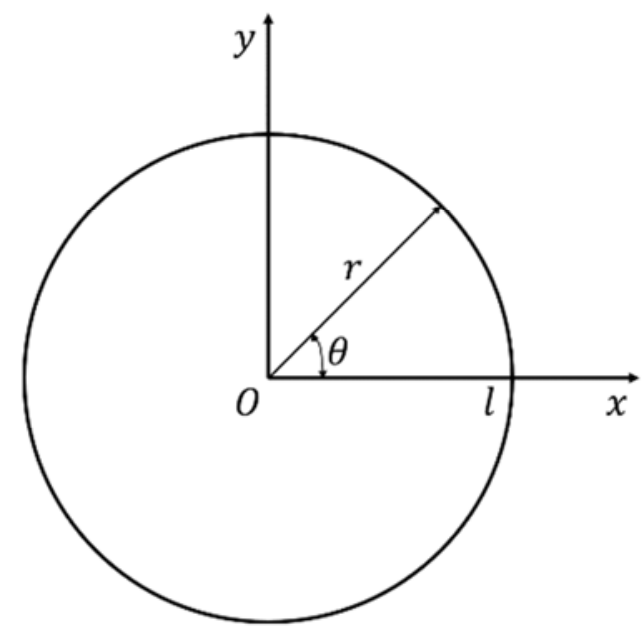

图2 固定边界的圆形薄膜。

$$
W(l, \theta, t)=0
$$

应用分离变量法, 设 $\mathrm{W}(r, \theta, t)=R(r) H(\theta) T(t)$, 代 入极坐标下的自由振动方程, 得到关于 $\mathrm{R}(r), \mathrm{H}(\theta)$, 和 $\mathrm{T}(t)$ 的三个常微分方程

$$
\begin{gathered}
r^{2} \frac{d^{2} R}{d r^{2}}+r \frac{d R}{d r}+\left(r^{2} \lambda^{2}-n^{2}\right) R=0,(0 \leq r<l) \\
\frac{d^{2} H}{d \theta^{2}}+n^{2} H=0,(0 \leq \theta<2 \pi) \\
\frac{d^{2} T}{d t^{2}}+T=0,(t>0)
\end{gathered}
$$

其中

$$
\lambda^{2}=\frac{\omega^{2}}{c^{2}}
$$

方程 (12) 的解为

$$
\mathrm{H}(\theta)=c_{1} \sin (n \theta+\varphi)
$$

将周期性条件 $\mathrm{W}(r, 0, t)=W(r, 2 \pi, t)$ 代入式 (15) , 可得 $\mathrm{n}$ 为整数。方程 (13) 的解为

$$
T(t)=c_{2} \sin (\omega t+\psi)
$$

方程 (11) 为n阶贝塞尔方程, 解为 [15]

$$
R(r)=c_{3} J_{n}(\lambda r)+c_{4} Y_{n}(\lambda r)
$$

其中, $J_{n}(\lambda r)$ 为第一类贝塞尔函数, $Y_{n}(\lambda r)$ 为第二类 贝塞尔函数。考虑到 $\mathrm{R}(0)$ 应该有界, $Y_{n}(0)=\infty$, 所以 $c_{4}=0$, 则式 $(17)$ 变为

$$
R(r)=c_{3} J_{n}(\lambda r)
$$
及

将式（18）代入边界条件（10）, 得到 $\mathrm{R}(l)=0$, 以

$$
J_{n}(\lambda r)=0
$$

记第 $\mathrm{m}$ 个正零点为 $\beta_{m}^{(n)}$, 则方程（19）的固有值为

$$
\lambda^{(n)}{ }_{m}=\beta_{m}^{(n)}
$$

将式（20）代入式（14）, 可得固有频率为

$$
\omega_{m, n}=\frac{\beta_{m}^{(n)}}{l} \sqrt{\frac{T}{\rho}}
$$

由式 (21) 可以看出, 等应力圆形薄膜的固有频率与 膜面张力正相关, 与膜面的半径负相关, 其他影响因素都 与膜材的基本参数有关。

基于以上理论推导, 本文设计了一种膜结构预张力振 动测试法: 控制膜面振动边界为圆形, 通过测量固定区域 的自振频率, 反推出膜面的张力。

\section{3. 膜结构预张力振动测试法试验}

\section{1. 试验设备}

\subsection{1. 膜材料}

本试验膜材料为PTFE膜材, 该膜材的基材为超细玻 璃纤维织物, 涂层为聚四氟乙烯树脂。其基本参数如下: 膜材密度 $1857 \mathrm{~kg} / \mathrm{m}^{3}$, 厚度 $0.70 \mathrm{~mm}[16]$ 。

\subsection{2. 测试设备}

试验设备有动态信号分析系统 (包括加速度传感器)、 拉力传感器、应变测试仪和本课题组搭建的试验平台等。 试验前需要对应变测试仪和拉力传感器进行标定, 使得拉 力值转化为应变测试仪读数, 标定的分度值为 $100 \mathrm{~N}$ 。试验 要求膜结构预张力逐级增加, 利用调节螺杆和夹具手动逐 级施加拉力。膜材按照经纬向裁剪成 $800 \mathrm{~mm} \times 800 \mathrm{~mm}$ 的矩 形, 两条相邻边上各安装 3 个拉力传感器。试验现场图如 图3所示。

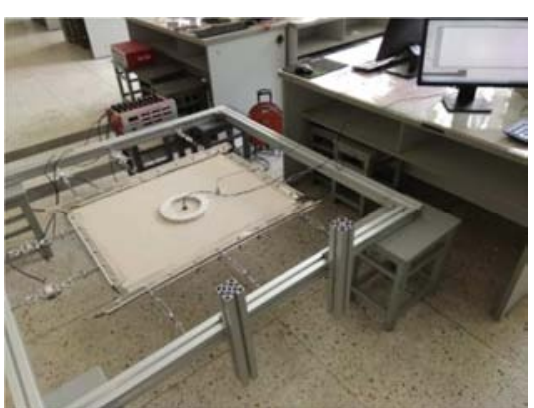

图3 试验现场图。 


\subsection{3. 固定边界设备}

膜面的自振频率与其边界条件有关，因此固定膜面测 量区域的边界条件成为振动法测量膜结构预张力的关键。 本试验设计了一种圆环形磁性夹具作为固定边界, 如图4 所示, 在金属圆环一侧开槽, 在槽内使用螺丝固定住 12 块磁性材料, 槽的深度与磁铁的厚度一致。图4中为磁性 夹具的下盘, 制作一块同样的金属圆环作为夹具的上盘, 上下两盘分别放置在膜的上下两面，利用磁铁之间的相互 作用, 使两块金属圆环紧密贴合, 从而控制膜面的振动边 界。

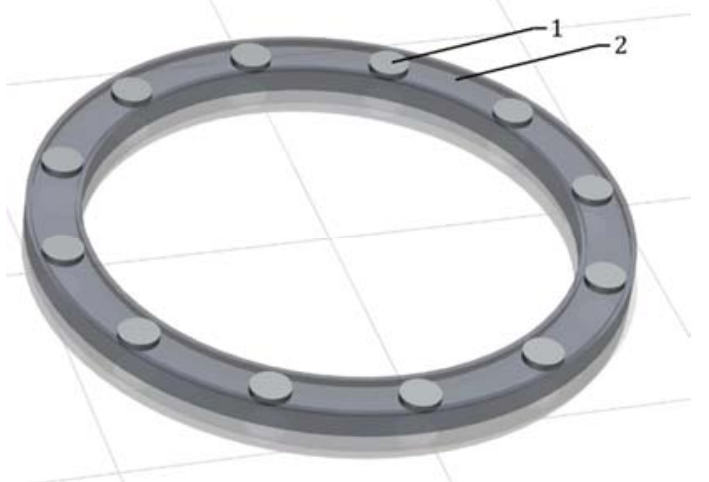

1-磁铁, 2-金属圆环

图4 磁性金属夹具。
金属圆环内径为 $180 \mathrm{~mm}$, 试验时上盘置于膜面上方, 下盘置于膜面下方。加速度传感器本身带有磁性, 利用磁 性将加速度传感器固定在圆环中心的膜面, 膜面振动时, 加速度传感器测得膜面振动数据, 传输至电脑分析软件, 最终得到圆环内膜面振动频率。

同时为验证圆环固定边界是否有效，在圆环外距外径 $90 \mathrm{~mm}$ 处固定一个加速度传感器。轻敲圆环内膜面, 环内 传感器能测得明显的主频, 但是环外的传感器无法捕捉到 主频, 表明圆环内的振动无法传递至圆环外, 由此验证磁 性金属圆环对膜面振动边界的固定的有效性。

\section{2. 试验步骤}

试验前先预加载10N以防止初始状态膜面松驰, 然后 再将应变仪清零。试验时逐级施加拉力, 施加拉力范围为 $300 \mathrm{~N} 2400 \mathrm{~N}$, 分度值为 $300 \mathrm{~N}$, 此时膜面张力为 $375 \mathrm{~N} / \mathrm{m} \sim 3000 \mathrm{~N} / \mathrm{m}$, 分度值为 $375 \mathrm{~N} / \mathrm{m}$ 。加载过程中取下圆 环的下盘, 加载至所需拉力后, 再将下盘放回膜面与上盘 组成固定边界, 以保证圆环内的张力与圆环外相同。固定 边界完成后, 轻敲圆环内的标志点, 圆环内加速度传感器 采集振动数据, 电脑分析软件得到圆环内膜面振动频率。 为观察张力增大时圆环的固定效果, 同时需要分析圆环外 加速度传感器的采集数据。为保证试验数据可靠性, 每次 加载采集三次数据取平均值。试验中圆环内传感器采集的 部分振动如图5所示。

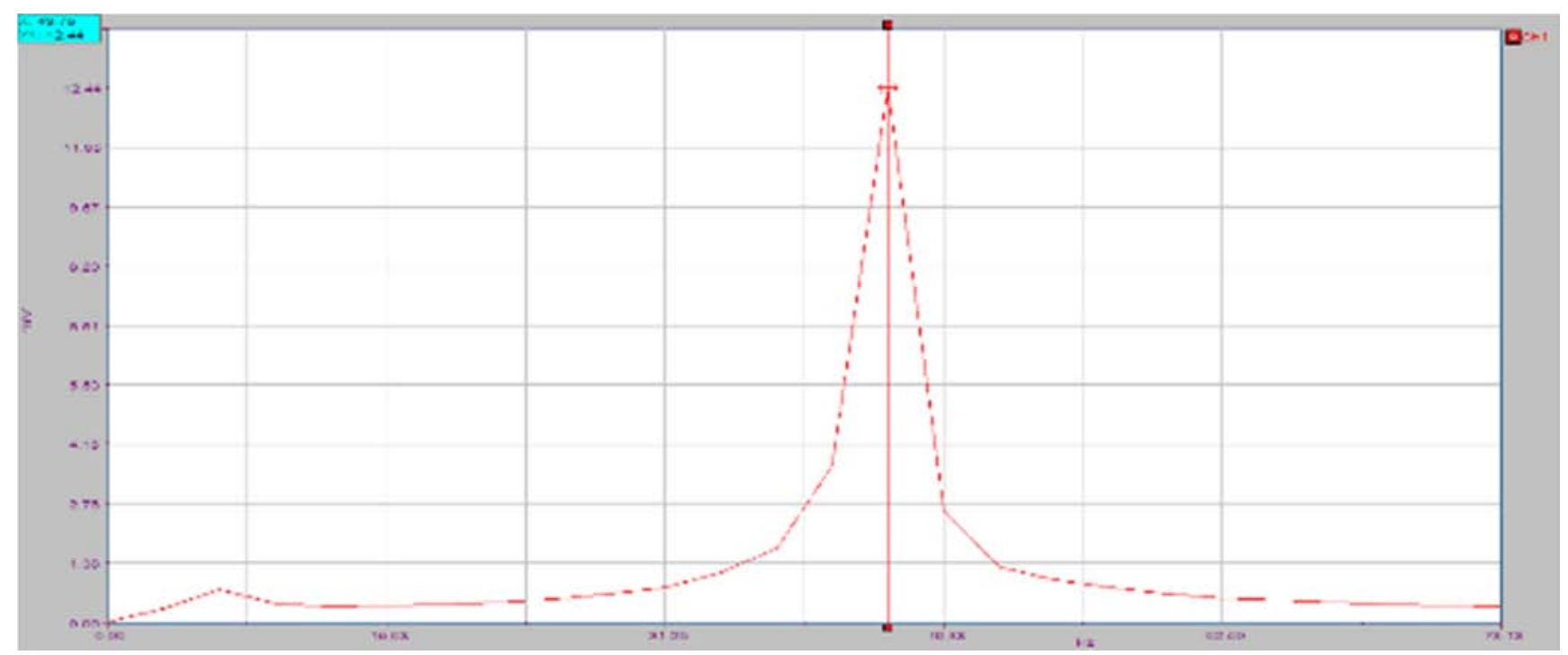

图5 圆环内振动频率图。

\section{3. 试验结果及分析}

图6是膜面张力逐级增大时圆环内外的振动频率对比 图, 结果表明: (1) 膜结构膜面张力与自振频率存在线 性关系。在较低的张力范围内, 随着膜面张力的增大, 膜 面自振频率呈较大幅度的线性增长。膜材加载至 $1500 \mathrm{~N} / \mathrm{m}$
后频率的增幅小于初期; （2）圆环能有效的控制膜面的 振动边界。圆环的传感器在膜面张力为 $3000 \mathrm{~N} / \mathrm{m}$ 之前均无 法采集到明显的振动主频, 膜面的振动被控制在圆环内。 验证了利用固定边界进行膜结构预张力振动法测试的可 行性。 


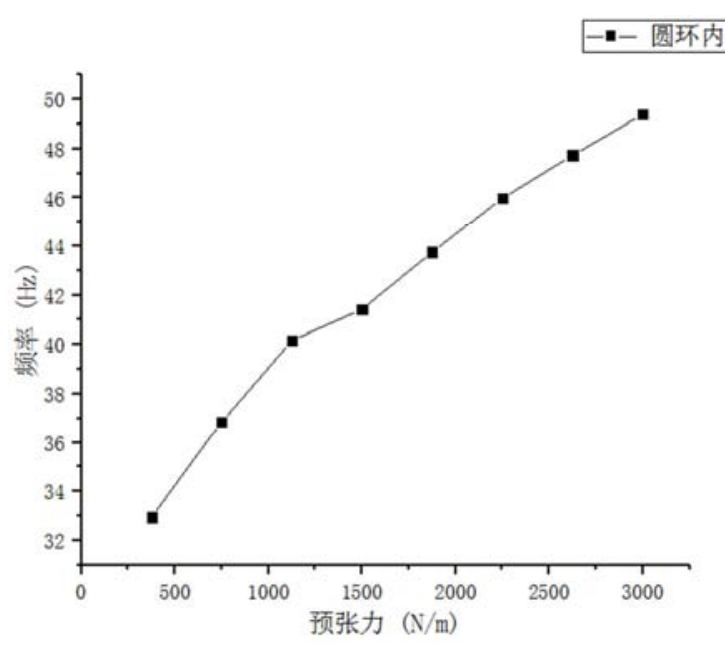

\section{4. 膜结构的数值仿真}

\section{1. 圆形膜结构的数值仿真}

为了检验固定边界后振动法测量膜面张力的准确性, 采用ANSYS软件进行数值计算。

在有限元软件中建立固定边界的正交各向异性圆形 薄膜结构模型, 先进行静力学分析, 完成膜结构预张力的 施加, 再进行模态分析, 可以确定膜结构的固有频率。选 用ANSYS壳单元SHELL181, 纬向弹性模量 $880 \mathrm{MPa}$, 经 向弹性模量 $1550 \mathrm{MPa}$, 密度 $1857 \mathrm{~kg} / \mathrm{m}^{3}$, 单元厚度 $0.0007 \mathrm{~m}^{[11]}$, 建立直径 $180 \mathrm{~mm}$ 的圆形薄膜模型, 进行模态 分析, 得到薄膜的各阶振动频率及振型。数值模拟得到的 圆形薄膜振动的各阶振型如图7所示。

图6 圆环内膜面振动频率。

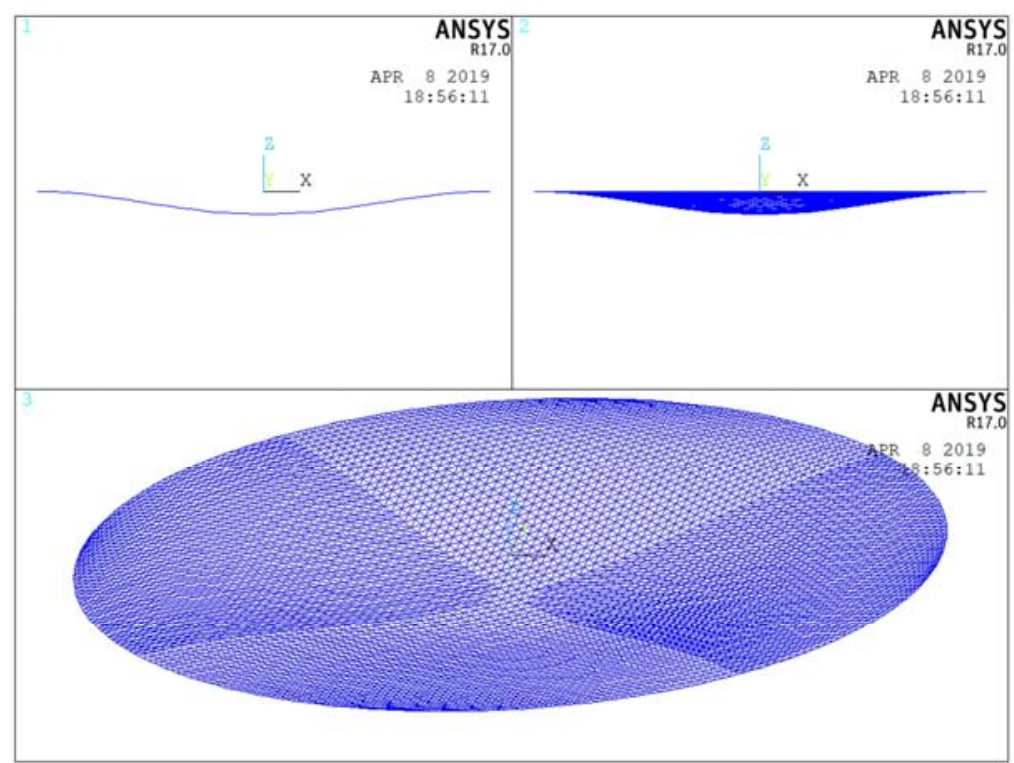

（a）一阶振型

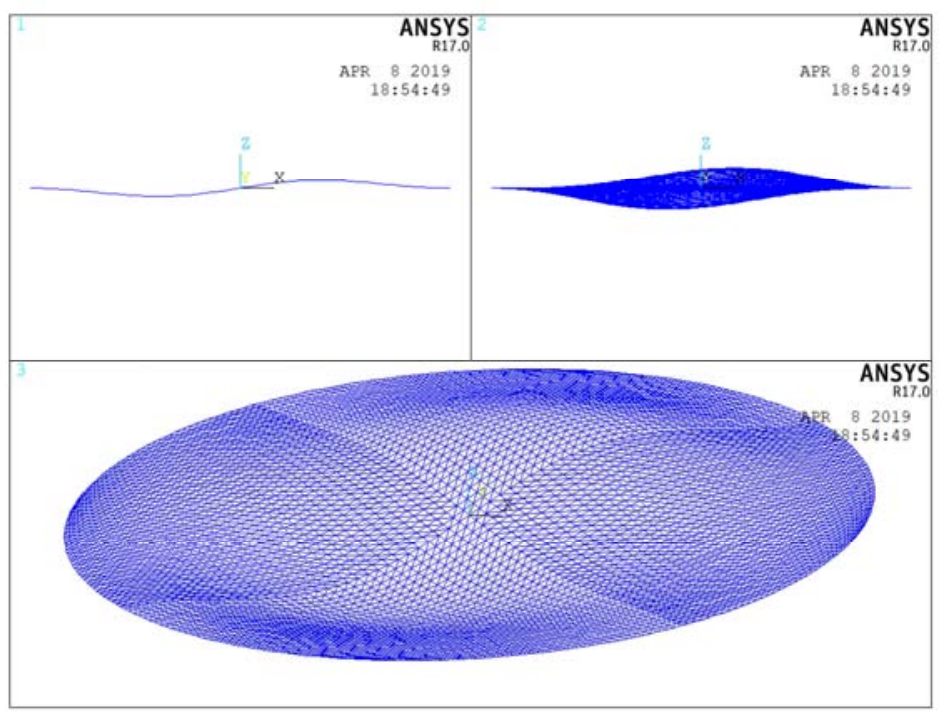

（b）二阶振型 


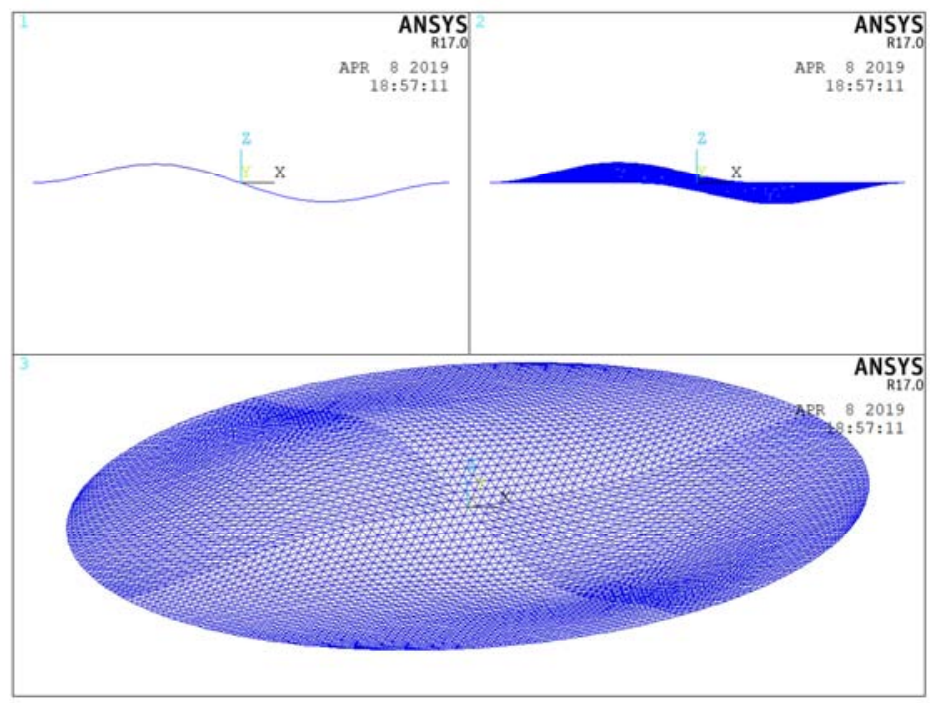

（c）三阶振型

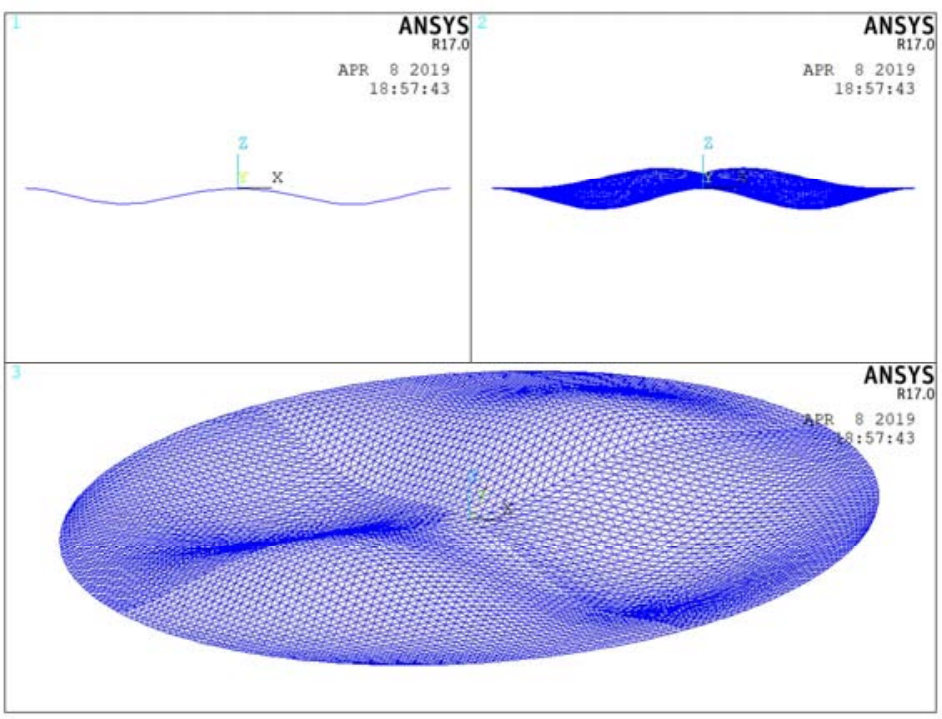

（d）四阶振型

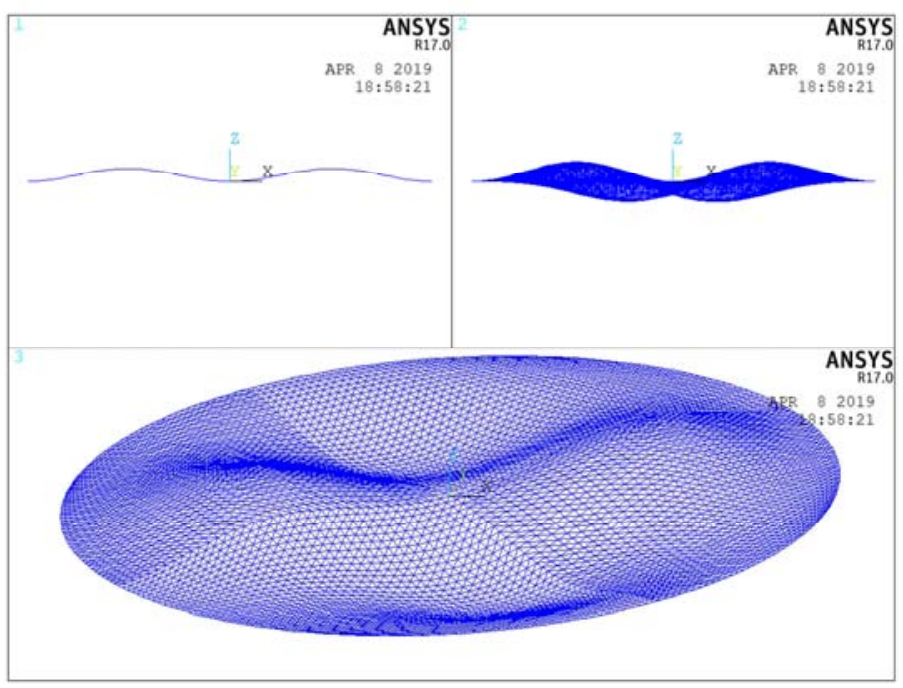

(e) 五阶振型 


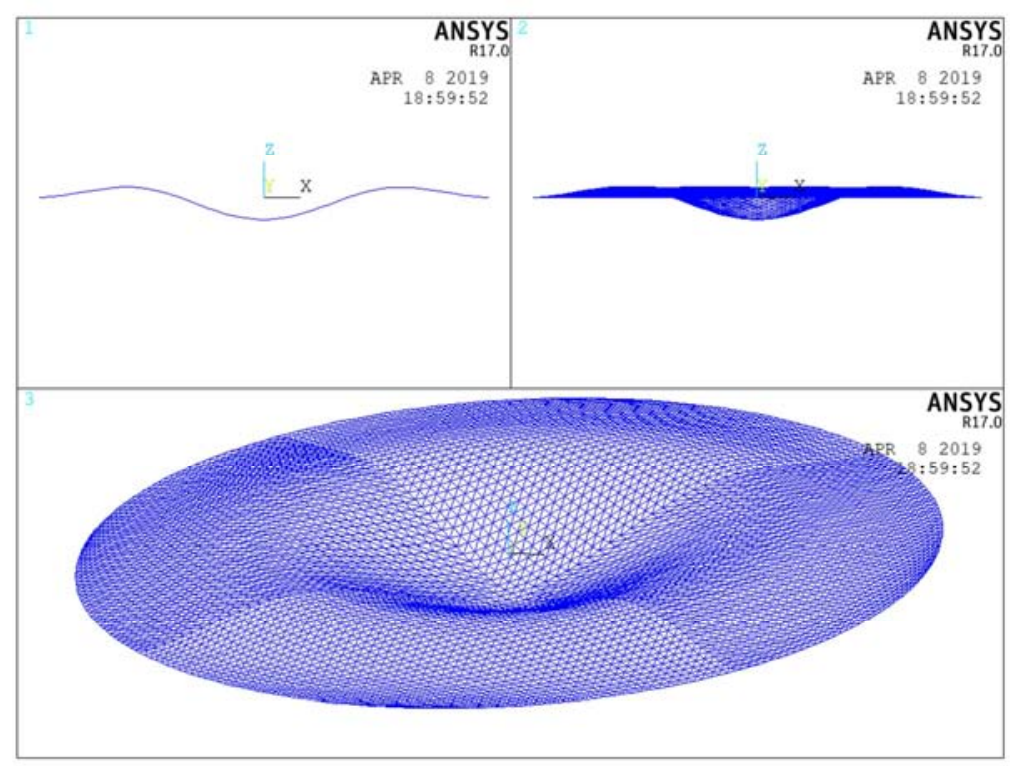

(f) 六阶振型

图7 圆形薄膜一至六阶振型。

\section{2. 数值模拟与试验结果分析}

不同张力下圆形膜面振动频率的数值模拟结果与试 验结果如表1所示。结果表明:（1）数值模拟结果和试验 吻合良好, 数值模拟是建立的圆形固定边界膜模型, 进一 步证明了固定边界振动法测量膜面张力的准确性。（2） 预张力小于 $800 \mathrm{~N} / \mathrm{m}$ 时, 试验测得频率低于数值模拟结果, 预张力在 $800 \mathrm{~N} / \mathrm{m} \sim 2400 \mathrm{~N} / \mathrm{m}$ 之间时, 数值模拟结果与试验 结果差值很小。预张力大于 $2400 \mathrm{~N} / \mathrm{m}$ 后, 随着预张力的增 大, 数值模拟结果仍然线性增长, 但是试验结果的增幅变 小, 原因是试验中随着预张力的增大, 圆环对膜面的固定 作用逐渐减弱, 此时测量区域的边界并不是圆环内的区域, 而是受到圆环外界的影响, 测量区域增大, 自振频率降低。

表1 不同张力下试验及数值模拟的膜面振动频率。

\begin{tabular}{lllll}
\hline 张力 $(\mathbf{N} / \mathbf{m})$ & $\mathbf{3 7 5}$ & $\mathbf{7 5 0}$ & $\mathbf{1 1 2 5}$ & $\mathbf{1 5 0 0}$ \\
\hline 试验频率 $(\mathrm{Hz})$ & 33.0 & 36.9 & 40.2 & 41.4 \\
数值模拟频率 $(\mathrm{Hz})$ & 35.2 & 37.5 & 40.5 & 42.5 \\
张力 $(\mathrm{N} / \mathrm{m})$ & 1875 & 2250 & 2625 & 3000 \\
试验频率 $(\mathrm{Hz})$ & 43.8 & 45.9 & 47.7 & 49.4 \\
数值模拟频率 $(\mathrm{Hz})$ & 44.8 & 47.0 & 49.4 & 51.7 \\
\hline
\end{tabular}

\section{5. 结论}

固定膜面的部分边界, 此边界内膜面自振频率只与膜 面张力有关, 这为振动法测量膜面张力提供了理论基础。

试验结果和数值模拟结果验证了此方法的可行性: 当 PTFE膜的膜面张力小于 $3000 \mathrm{~N} / \mathrm{m}$ 时, 膜面张力逐渐增大, 本方法能较准确地测量出膜面的实时张力。当膜面张力很 大时, 本试验的固定设备对边界的固定作用逐渐失效, 不 难推理出, 选用固定能力足够的设备, 能够使固定失效张 力大于膜材的撕裂张力, 从而扩大测量范围, 提高测量精 确度。
工程测试中对不同种类的膜材, 只要对膜材小试样进 行固定边界振动测量, 完成张力-频率的标定, 就可以对 各种工况下的膜材张力进行有效的测量, 完全符合工程实 际中的操作简单, 适用性广和测量精度高的要求, 有着广 阔的应用前景。

\section{参考文献}

[1] 张其林. 索和膜结构 [M]. 上海: 同济大学出版社, 2002

[2] 张其林.膜结构在我国的应用回顾和未来发展 [J].建筑结 构,2019,49(19):55-64.

[3] Liu Changjiang, Deng Xiaowei , Liu Jian, Zheng Zhoulian . Impact-induced nonlinear damped vibration of fabric membrane structure: Theory, analysis, experiment and parametric study [J]. 2019. 159: 389-404.

[4] 魏德敏,杨桂通.冲击荷载下弹粘塑性矩形薄板的动力响应 [J].工程力学.1986. 3(3).20-32

[5] 邓梁波,叶天麒.圆板在物体撞击下的非线性动力响应[J].力 学学报.1990,22(4),420-428

[6] 江松青, 李永池. 横向冲击荷载下加筋板的非线性动力响 应[J].中国科学技术大学学报.2000.30(4).406-413

[7] 李阳. 建筑膜材料和膜结构的力学性能研究与应用[D]. 上 海：同济大学,2007.

[8] 孙战金.膜结构预张力测定技术简述 [A].天津大学.第四届全 国现代结构工程学术研讨会论文集 [C].天津大学:全国现代 结构工程学术研讨会学术委员会,2004:3:496-498

[9] 孙战金. 建筑膜材预张力值测试的理论和实验研究 [D]. 上海: 同济大学,2004. 
[10] 麻海燕,单建.建筑膜结构荷载的超声快速测定方法 [J].工业 建筑,2006(09):77-80.

[11] 刘长江.建筑膜结构非线性振动及其预张力测量理论和试 验研究[D].重庆: 重庆大学,2012.

[12] 余志祥,赵雷.张拉膜结构自振特性研究[J].西南交通大学学 报,2004(06):734-739.

[13] 吴福光,蔡承武,徐兆.振动理论 [M]. 北京: 高等教育出版 社, 1987:233-241
[14] 陈才生,李刚,周继东,王文初.数学物理方程[M].北京：科学 出版社,2008:233-255

[15] 林文静,陈树辉,李森. 圆形薄膜自由振动的理论解 [J]. 振动与 冲击,2009,28(05):84-86+206.

[16] 曹志威. 基于数字图像相关法的建筑用膜力学性能研究[D]. 南京理工大学,2018. 